Article

\title{
Synthesis of Framework Isomer MOFs Containing Zinc and 4-Tetrazolyl Benzenecarboxylic Acid via a Structure Directing Solvothermal Approach
}

\section{Carlos Ordonez ${ }^{1, *}$, Tiffany L. Kinnibrugh ${ }^{1, \dagger}$, Hongwu Xu ${ }^{2}$, Jennifer Lindline ${ }^{3, \dagger}$, Tatiana Timofeeva ${ }^{1, \dagger}$ and Qiang Wei ${ }^{1, \dagger}$}

1 Department of Biology and Chemistry, New Mexico Highlands University, Las Vegas, NM 87701, USA; E-Mails: tkinnibr@aps.anl.gov (T.L.K.); tvtimofeeva@nmhu.edu (T.T.); qwei@nmhu.edu (Q.W.)

2 Los Alamos National Laboratory, Los Alamos, NM 87545, USA; E-Mail: hxu@lanl.gov

3 Department of Natural Resources Management, New Mexico Highlands University, Las Vegas, NM 87701, USA; E-Mail: lindlinej@nmhu.edu

$\dagger$ These authors contributed equally to this work.

* Author to whom correspondence should be addressed; E-Mail: cordone1 @ live.nmhu.edu; Tel.: +1-609-412-9231.

Academic Editor: Lan Li

Received: 29 November 2014 / Accepted: 16 February 2015 / Published: 2 April 2015

\begin{abstract}
The solvothermal synthesis of framework isomers was carried out using the hybrid carboxylate and tetrazolate functional ligand, 4-tetrazolyl benzenecarboxylic acid $\left(\mathrm{H}_{2} \mathrm{TBC}, \mathrm{TBC}=4\right.$-tetrazolyl benzenecarboxylate $)$ and zinc. $\mathrm{H}_{2} \mathrm{TBC}$ was also synthesized with the solvothermal approach, and is referred herein as structure 1. Using single-crystal X-ray diffraction, we found that the tetrazolate groups of TBC show an unusual "opposite-on" coordination mode with zinc. Three previously characterized metal-organic frameworks (MOFs) were obtained by systematically changing the solvents of the $\mathrm{H}_{2} \mathrm{TBC}-\mathrm{Zn}$ reaction, (1) ZnTBC, 2, which has a non-porous structure; (2) $\mathrm{Zn}_{2}(\mathrm{TBC})_{2}\left(\mathrm{H}_{2} \mathrm{O}\right)$, 3, which has an amphiphilic pore structure and (3) $\mathrm{Zn}_{2}(\mathrm{TBC})_{2}\{$ guest $\}$, 4, which is porous and has channels containing uncoordinated $\mathrm{N}$ heteroatoms. Fluorescence spectra of $\mathbf{4}$ reveal a strong blue emission mainly from the TBC ligands.
\end{abstract}


Keywords: hybrid ligand; tetrazolate; opposite-on coordination; solvothermal technique

\section{Introduction}

Metal-organic frameworks (MOFs) have recently become active materials in gas storage, and separation applications because of their high surface area, functionalizable pore structures and diverse coordination modes between ligands and metal ions [1-6]. The combination of carboxylate and zinc ions is the most common coordination pair in the reported MOFs. Recently, new ligands based on nitrogen coordination functional groups with zinc ions have become popular due to their similar pKa values of about 4 , and their multiple electron donating positions [7,8]. New studies are being performed on hybrid ligands that incorporate two or more functional groups to determine if more topologies and applications can result. The most studied examples of hybrid ligands are those that incorporate the tetrazolate and carboxylate functional groups attached to a benzene ring formally known as the tetrazolyl benzenecarboxylic acid $\left(\mathrm{H}_{2} \mathrm{TBC}\right)$ (Figure 1).

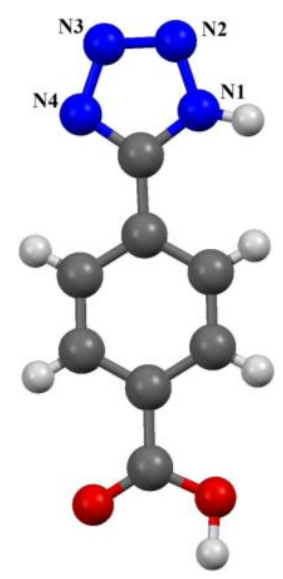

Figure 1. The structure of the $\mathrm{H}_{2} \mathrm{TBC}$ ligand determined by X-ray single crystal analysis, $\mathbf{1}$. (C: dark gray, $\mathrm{N}$ : blue, $\mathrm{O}$ : red and $\mathrm{H}$ : light gray).

One of the most difficult steps in engineering MOFs is finding the correct synthetic parameters that will yield the desired structure. Parameters such as the temperature, pressure, $\mathrm{pH}$, heating time, metal source, organic ligands and solvents used are vital towards producing structures with high purity, crystal size, and particular properties [9]. The hydrothermal technique is one of the most widely used forms of synthesis for MOFs because it utilizes readily available water as a solvent source. While this technique is very handy at producing water stable MOFs, other water-unstable MOFs require other synthetic approaches. The solvothermal synthesis is the second most used technique for the preparation of MOFs with different types of topologies and porosities. The solvothermal technique offers the opportunity of utilizing other solvent sources with different polarities, molecular sizes, and properties that the hydrothermal technique lacks, plus the prospect of forming new organic ligands not possible with regular synthetic conditions [10]. On occasion, changing the solvent system or the reaction conditions to obtain MOFs can result in MOFs built from the same metal ions and ligands, but differ in their connectivity or structure all together. This phenomenon has been described by Makal, Yakovenko, and Zhou (2011) as 
"Framework Isomers" [11]. They define "Framework Isomers" as "MOFs constructed from the same ligands and metal species that display different network structures". This type of phenomenon has been shown in MOFs constructed from $\mathrm{H}_{2}$ TBC and Zinc by changing the solvent system. Historically MOFs utilizing $\mathrm{H}_{2}$ TBC as the ligand have for the most part been paired with zinc via the hydrothermal technique. Li et al. (2008) [12] reported the synthesis of a zinc(II) 4-(5H-tetrazolyl)benzoic coordination polymer exhibiting a GSI topology. This particular MOF was produced in a one pot hydrothermal synthesis set at $140{ }^{\circ} \mathrm{C}$ utilizing sodium 4-cyanobenzoic acid, sodium azide, and zinc bromide as the starting materials. The article stated that the addition of $\mathrm{HCl}$ will cause decomposition of the MOF into the $\mathrm{H}_{2} \mathrm{BTC}$ ligand, and production of the MOF from the $\mathrm{H}_{2} \mathrm{BTC}$ ligand and zinc bromide was not observed. Another paper by Song et al. (2008) [7] also reported the same structure as that as Li et al. (2008) [12], but with Co ${ }^{\text {II }}$ doping applied to the synthesis. Similar to Li et al. (2008) [12], Song et al. (2009) [7] utilized 4-cyanobenzoic acid, sodium azide, zinc chloride, and cobalt chloride to produce the MOF, however the hydrothermal technique applied temperature of $160{ }^{\circ} \mathrm{C}$ with a heating period of two days. A second paper by Li et al. (2011) [8] reported the synthesis of a tubular framework constructed by trigonal rings second building units. While this paper did not report specifically the details of the synthesis, it is known that a hydrothermal technique was utilized on sodium azide, sodium 4-cyanobenzoic acid and zinc bromide, according to the procedures by Li et al. (2008) [12], except maybe the heating conditions. Wei et al. (2012) [13] described the synthesis of a new highly porous $\mathrm{Zn}$-TBC structure prepared via the solvothermal technique. Unlike the previous reported $\mathrm{Zn}-\mathrm{TBC}$ structures, the structure presented by Wei et al. (2012) was synthesized with zinc nitrate and the $\mathrm{H}_{2} \mathrm{TBC}$ ligand dissolved in methanol at a temperature of $150{ }^{\circ} \mathrm{C}$ for three days. This achievement prompted the utilization of the solvothermal technique to produce several MOFs that might realize different topologies by varying the solvent system in the synthetic conditions but maintaining the heating conditions and starting materials constant. This study reports a crystallographic study of solvothermally grown $\mathrm{H}_{2} \mathrm{TBC}$ ligands, as well as framework isomer MOFs composed of the 4-tetrazolyl benzenecarboxylic acid $\left(\mathrm{H}_{2} \mathrm{TBC}\right)$ ligand and zinc metal ions under varying solvent systems. When the pyridyl group is replaced by the tetrazole, the multi-coordination modes of tetrazole groups [14] of the TBC ligand are expected to increase variations of link directions and help to build multi-topology MOF structures. In the synthesized MOFs, the "opposite-on" [15] coordination site of tetrazolate groups with zinc was the main coordination mode. Such coordination mode therefore prevented the formation of zinc clusters, leading the MOFs to the condensed form. However, the MOF with both hydrophilic and hydrophobic channels, and the MOF with channel structures containing uncoordinated $\mathrm{N}$ heteroatoms were obtained by changing the reaction solvents used.

\section{Results and Discussion}

\subsection{An "Imidazolate"-Like Coordination Mode for Tetrazolate in TBC}

Addition of excess $\mathrm{Zn}\left(\mathrm{NO}_{3}\right)_{2} \cdot 6 \mathrm{H}_{2} \mathrm{O}$ during a reaction with $\mathrm{H}_{2} \mathrm{TBC}$ under hydrothermal conditions yielded a quantitative amount of hair-like crystals of $\mathbf{1}$. To have a more clear understanding of the coordination behaviors of the TBC ligands, the arrangement of the proton ion on the TBC ligands were studied by single X-ray crystallography test of the crystalized TBC ligand only. As shown in Figure 1, 
the crystal structure of the TBC ligands, demonstrates that the proton is located at $\mathrm{N}(1)$ positions, indicating that the electrons were more localized around $\mathrm{N}(1)$ or $\mathrm{N}(4)$ atoms. The distances of $\mathrm{N}-\mathrm{N}$ in $\mathrm{N}(1)-\mathrm{N}(2)$ is 1.353 (2) $\AA$ and N3-N4 is 1.335 (2) $\AA$, which indicated the $\mathrm{N}$ to $\mathrm{N}$ single and double bonding character respectively elongated the tetrazolate rings. This result is consistent with the observation in the TBC-Zn coordination compounds. Studies of the bonding distances show the distance of $\mathrm{N}(2)-\mathrm{N}(3)$ is about 1.306 (2) $\AA$ (average value from CCDC is $1.317 \AA$ ) which is close to the $\mathrm{N}=\mathrm{N}$ double bond, and $\mathrm{N}(1)-\mathrm{N}(2)$ or $\mathrm{N}(3)-\mathrm{N}(4)$ is about 1.350 (2) $\AA$ (average value from CCDC is $1.346 \AA$ ) which is more close to the $\mathrm{N}-\mathrm{N}$ single bonds [16]. Therefore, it suggests that in the deprotonated tetrazolate rings, there is an aromatic effect and the electron is delocalized around the whole ring. The $\mathrm{N}-\mathrm{N}$ distances suggested that between $\mathrm{N}(2)$ and $\mathrm{N}(3)$ the electron overlap is stronger than the rest of the ring. As a result, there was a higher amount of electron density around $\mathrm{N}(1)$ and $\mathrm{N}(4)$ than that of $\mathrm{N}(2)$ and N(3). Though N(1) and N(4) had more steric effect in coordination, they seem to be more easily coordinated to the zinc ions than $\mathrm{N}(2)$ and $\mathrm{N}(3)$ in these 3-D frameworks because more donor electrons are around them.

It is also found that in the reported tetrazolate ligands-metal compounds [12], usage of $N(1)$ or $N(4)$ atoms to mono-coordinate with metal ions was a general coordination mode, which also indicated the electron influence to the coordination mode of such ligands. If the $\mu_{2}-\mathrm{N}(2), \mathrm{N}(3)$ coordination mode of tetrazolate is analogous to the carboxylate group, the "opposite-on" $\mu_{2}-\mathrm{N}(1), \mathrm{N}(4)$ coordination mode of tetrazolate is the analogous to imidazolates. These results were also confirmed by quantum chemical calculations of charge distribution within the molecule. Figure 2 shows that N(1) and N(4) have more negative charge as compared to $\mathrm{N}(2)$ and $\mathrm{N}(3)$. In fact both oxygen atoms, $\mathrm{N}(1)$ and $\mathrm{N}(4)$ appeared to contain the same amount of negative charge that allowed for coordination with the zinc ion. As it will be discussed below reaction between $\mathrm{Zn}\left(\mathrm{NO}_{3}\right)_{2} \cdot 6 \mathrm{H}_{2} \mathrm{O}$ with $\mathrm{TBC}$ in the different solvent systems results in different MOF structures from nonporous to porous. The solvate molecules here act as structure directing agents during the formation of MOFs 2-4. However, in all of MOFs 2-4, coordination modes of the tetrazolate group of TBC were the same. Both $\mathrm{N}(1)$ and $\mathrm{N}(4)$ atoms were fully coordinated to zinc. In all of the frameworks 2,3 and $4, \mathrm{~N}(1)$ and $\mathrm{N}(4)$ were preferable coordination positions.

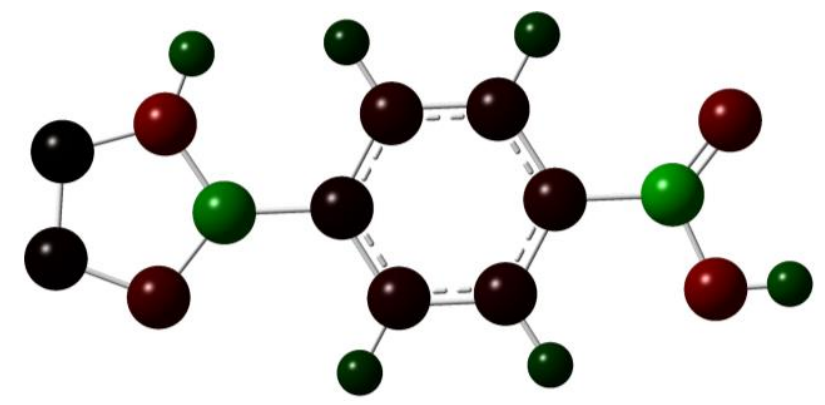

Figure 2. Computational analysis of TBC ligand. The color demonstrates the charge distribution within the molecule. The red indicates a negative charge, green a positive charge, and black a neutral charge. 


\subsection{Synthesis and Characterization of $\mathrm{ZnTBC}, 2, \mathrm{Zn}_{2}(\mathrm{TBC})_{2}\left(\mathrm{H}_{2} \mathrm{O}\right), 3$ and $\mathrm{Zn}_{2}(\mathrm{TBC})_{2}, 4$}

Reaction between $\mathrm{Zn}\left(\mathrm{NO}_{3}\right)_{2} \cdot 6 \mathrm{H}_{2} \mathrm{O}$ and $\mathrm{H}_{2} \mathrm{TBC}$ under solvothermal conditions in water gave a quantitative yield of colorless to yellowish crystals characterized as ZnTBC, framework 2 shown in Figure $3[7,12,17]$. In this framework, zinc atoms had tetrahedral coordination with two oxygen atoms from the carboxylate groups and two nitrogen atoms from the tetrazolate groups of the TBC ligands. The carboxylate groups bridge the zinc atoms to form chain structures, and such chains were linked by the tetrazolates to form a 3D network. The bridging carboxylate groups were in syn-anti conformation. Framework $\mathbf{2}$ is non-porous. Something to note in framework $\mathbf{2}$, is the TBC ligands only use N(1) and $\mathrm{N}(4)$ nitrogen atoms of the tetrazolate groups to coordinate with zinc. Unlike previous reports [12], framework 2 was synthesized using the $\mathrm{H}_{2}$ TBC ligand and not just sodium azide, and sodium 4-cyanobenzoic acid.

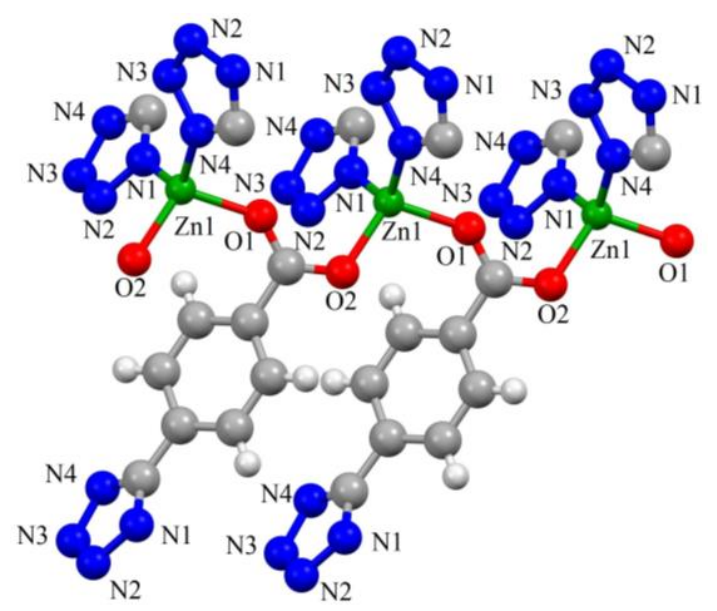

(a)

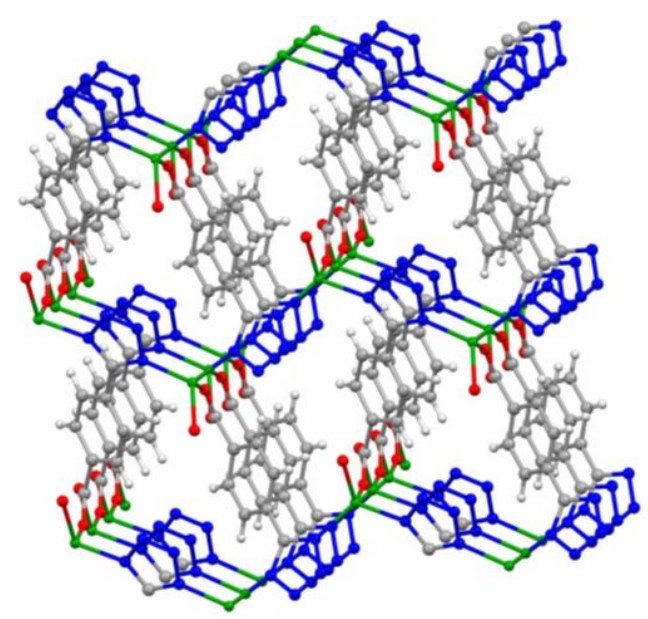

(b)

Figure 3. (a) The coordination environment of the zinc ions in compound 2. Symmetry codes, A: $x, y+1, z ; \mathrm{B}: x,-y+1, z-1 / 2 ; \mathrm{C}: x-1, y, z ; \mathrm{D}: x,-y+1, z+1 / 2 ; \mathrm{E}: x+1, y, z$; $\mathrm{F}: x, y-1, z ;(\mathbf{b})$ crystal packing structure. (Zn: green, $\mathrm{C}$ : gray, $\mathrm{N}$ : blue, $\mathrm{O}$ : red and $\mathrm{H}$ : white).

When synthesized in $\mathrm{H}_{2} \mathrm{O}$ and $\mathrm{MeOH}$ mixed solvents $(1: 1 \mathrm{v} / \mathrm{v})$, PXRD analysis showed a main product of compound $\mathbf{2}$ obtained but with a minor peak of an unknown phase which we will discuss in more detail later in the paper. Single crystal analysis of chosen crystals revealed the unknown phase may belong to a new framework structure $\mathrm{Zn}_{2}(\mathrm{TBC})_{2}\left(\mathrm{H}_{2} \mathrm{O}\right)$, compound 3 as seen in Figure 4.

Compound $\mathbf{3}$ had different coordination modes of the tetrazolate and carboxylate groups from 2 . In compound 3, the tetrazolate groups still used N(1) and N(4) atoms to coordinate with zinc ions, and carboxylate groups were bridged-coordinated with two zinc atoms/ions. There were two unique zinc coordination environments found in $3: \mathrm{Zn}(1)$ had a tetrahedral coordination environment bound to four TBC ligands, with two of the TBC ligands bound through the carboxylate carbonyl and two bound through the tetrazole $\mathrm{N}(1)$. This $\mathrm{Zn}(1)$ moiety formed an aggregated cyclic trimer $\mathrm{Zn}_{3}(\mathrm{TBC})_{6}$ (Figure 4a). The final network was formed by assembling the $\mathrm{Zn}_{3}$ trimers though $\mathrm{Zn}$ (2) atoms in a five coordinated trigonal bipyramid fashion through the tetrazolate groups. Hydration water molecules were found coordinated to the $\mathrm{Zn}(2)$ atoms. 


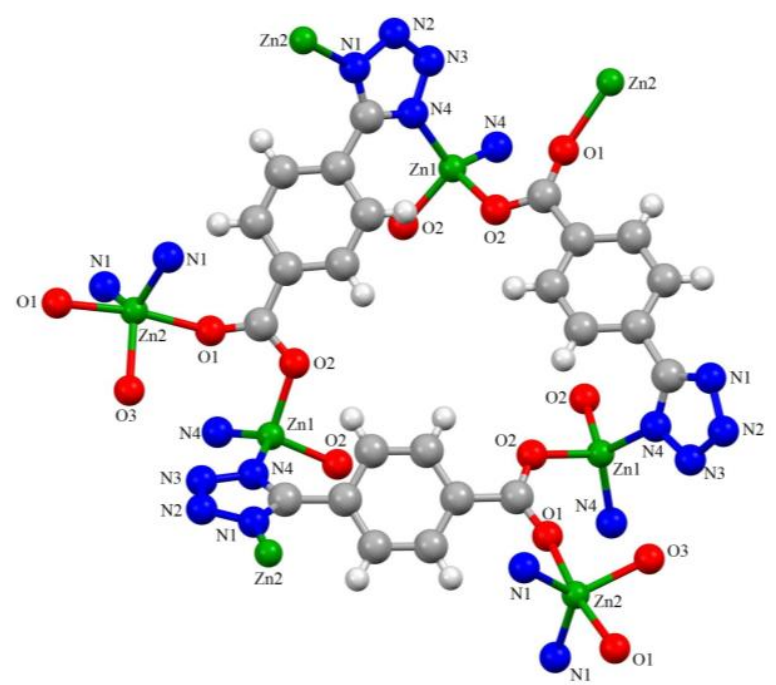

(a)

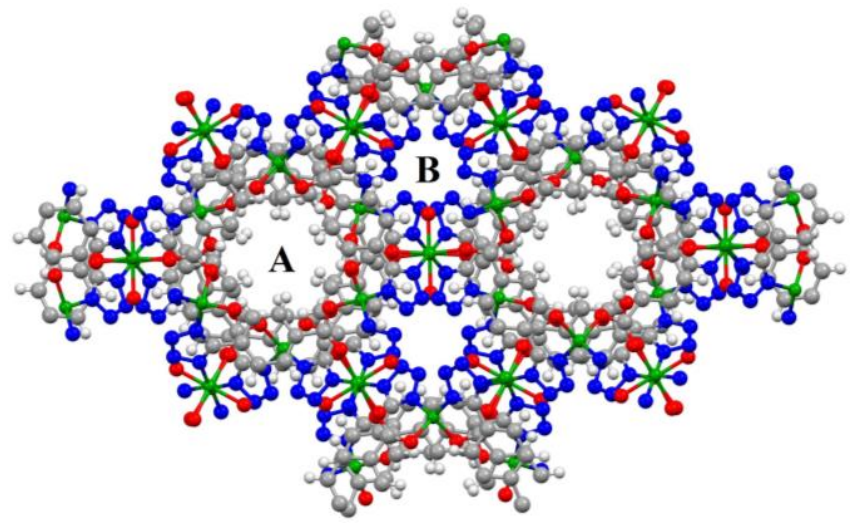

(b)

Figure 4. (a) The coordination modes of $\mathbf{3}$; (b) The channel structures in $\mathbf{3}$. Symmetry codes, $\mathrm{A}: x-y+1 / 3,-y+2 / 3,-z+13 / 6 ; \mathrm{B}:-x+2 / 3,-x+y+1 / 3,-z+11 / 6$; $\mathrm{C}:-x+y+1 / 3, y-1 / 3, z+1 / 6 ; \mathrm{D}:-y+1 / 3,-x+2 / 3, z+1 / 6 ; \mathrm{E}:-x+y+2 / 3, y+1 / 3$, $z-1 / 6 ; \mathrm{F}:-y+2 / 3,-x+1 / 3, z-1 / 6$. (Zn: green, $\mathrm{C}$ : gray, $\mathrm{N}$ : blue, O: red and H: white).

Unlike compound 2, compound $\mathbf{3}$ showed a 1D channels structure (Figure 4b). There are two types of channels structures that co-exist in $\mathbf{3}$, here referred to as Channel A and Channel B [8]. Channel A formed only by aromatic phenyl rings (shown as grey color in Figure 4), therefore the interior wall of Channel A was apparently hydrophobic in character. In contrast, the channel B had an interior wall composed by tetrazole nitrogen atoms (shown as a blue color in Figure $4 \mathrm{~b}$ ) and coordinated water molecules facing the channel suggesting that channel B was significantly hydrophilic in character. The maximum aperture size in Channel $\mathrm{A}$ is $\sim 1.3 \AA$ in diameter.

In the formation of $\mathbf{3}$, the solvents, $\mathrm{MeOH}$, and water seemed combinative to act as a template to induce the formation of the amphiphilic channel structures. An effort to produce pure $\mathbf{3}$ samples was unsuccessful. All of the reactions using water and $\mathrm{MeOH}$ mixed solvents 1:1 resulted in compound 2 as the main product. However, when anhydrous $\mathrm{MeOH}$ was applied as the solvent, a third new framework 4 resulted. As shown in Figure 5, in framework 4 each $\mathrm{Zn}$ atom was also tetrahedral coordinated with $\mathrm{N}_{2} \mathrm{O}_{2}$ set from the TBC ligands. However, different from 2 , the bridging carboxylate groups were in a syn-syn conformation, and isolated $\mathrm{Zn}_{2}(\mathrm{OOC})_{2}$ dimer structures formed in $\mathbf{4}$, which act as secondary building units and form a framework with channels along the $a$-axis. It was found that disordered methanol and/or water solvate molecules are located in those channels [13]. It was clear that during the formation of 4, $\mathrm{MeOH}$ molecules acted as structural directing agents. We previously reported that when the $\mathrm{MeOH}$ solvate was removed from the channels, a porous structure with a maximum BET surface area of $270 \mathrm{~m}^{2} \cdot \mathrm{g}^{-1}$ after thermal activation above $200{ }^{\circ} \mathrm{C}$ was found [13]. Further interest in the BET analysis, and sorption and desorption curves of this compound can be found in more details in our previous paper.

TBC ligands in 2 connect four $\mathrm{Zn}(\mathrm{II})$ atoms through $\mathrm{N}(1)$ and N(4) nitrogen atoms from tetrozolate groups and two syn-anti $\mathrm{O}$ atoms from carboxylate group. Each $\mathrm{Zn}$ (II) was four-coordinated by four TBC ligands. In the analysis of the topology of $\mathbf{2}$, the TBC ligands can be treated as 4-connecting 
building units in $\mathbf{2}$, and the structure of $\mathbf{2}$ therefore contained two types of 4-connecting nodes ( $\mathrm{Zn}^{2+}$ and TBC). In MOF 4 (Figure 5), TBC ligands bridged the $\mathrm{Zn}(\mathrm{II})$ ions through carboxylate groups in a syn-syn conformation to form isolated $\mathrm{Zn}_{2}$ clusters. In analysis of the topology of $\mathbf{4}$, such $\mathrm{Zn}_{2}(\mathrm{TBC})_{2}$ dimers were secondary building units tetrahedrally connected by 1,4-N atoms from two tetrazolate groups. Therefore, MOF 4 contained only one type of 4-connected node ( $\mathrm{Zn}_{2}$ dimer). Both $\mathbf{2}$ and $\mathbf{4}$ belong to 4-connected $6^{6}$-net topology. However, the larger secondary building units of $\mathrm{Zn}_{2}(\mathrm{TBC})_{2}$ resulted in a porous structure as MOF 4, while the distorted tetrahedral coordinated TBC as secondary building units in MOF $\mathbf{2}$ only generated a non-porous framework. TOPOS analysis revealed a GSI net for $\mathbf{1}$ and a gis net for 4. Efforts of using bulkier solvents, such as ethanol and isopropanol, to introduce new frameworks structures of the Zn-TBC system were not as successful. Only fine crystalline or gel like powders were obtained. Table 1 bellow compares the crystallographic data obtained for the organic ligand as well as the three MOFs obtained in this study.

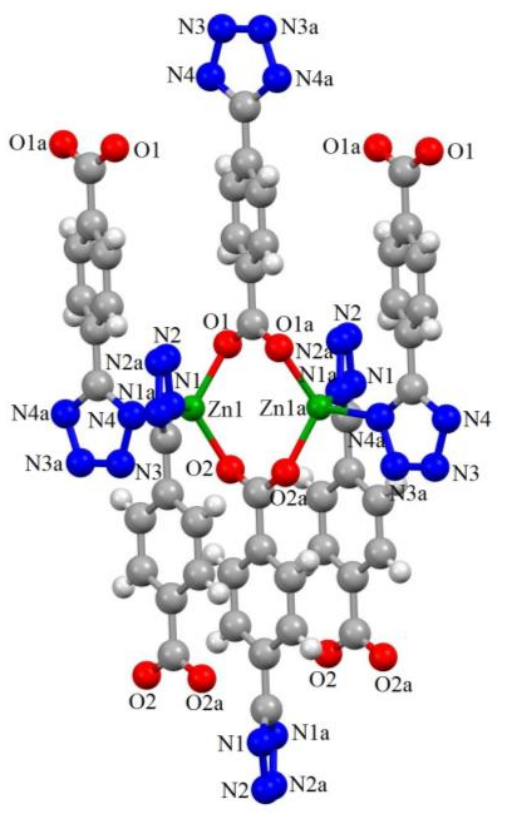

(a)

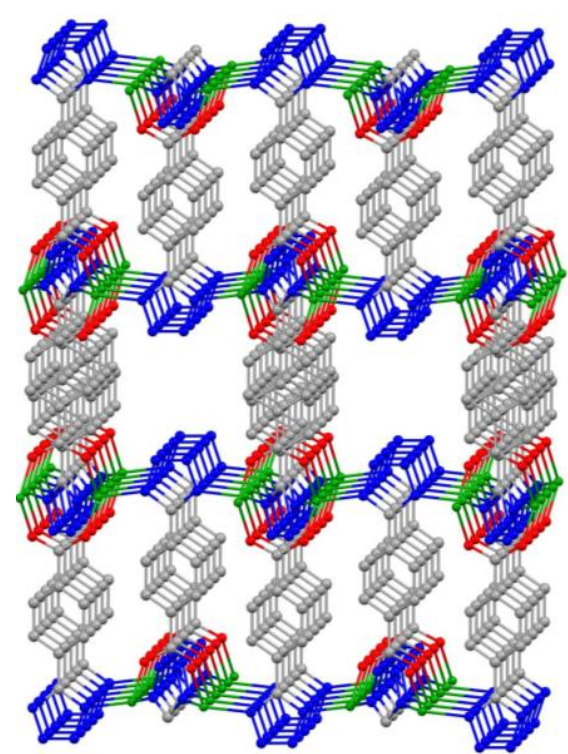

(b)

Figure 5. (a) Coordination of $\mathrm{Zn}$ and TBC ligands in 4. Symmetry codes, $\mathrm{A}:-x, y,-z-1 / 2$; $\mathrm{B}:-x+1, y,-z+1 / 2 ; \mathrm{C}:-x+1, y,-z-1 / 2 ; \mathrm{D}:-x+1,-y,-z ; \mathrm{E}:-x+1 / 2,-y+1 / 2,-z$; (b) The Channels structures along a-axis. Hydrogen atoms are omitted for clarity (Zn: green, C: gray, $\mathrm{N}$ : blue, O: red).

Powder X-ray Diffraction (PXRD) analysis of compound $\mathbf{1}$ showed excellent crystallinity for compound $\mathbf{1}$ as well as a good correlation to the simulated pattern obtained from the single crystal X-ray data sets as can be observed in Figure $\mathrm{S} 1$ in the supplementary data. As mentioned above, synthesis utilizing $\mathrm{H}_{2} \mathrm{O}$ and $\mathrm{MeOH}$ resulted in high quantities of compound 2, and a very minor amount of compound 3 (Figure S2). We believed that the equipment we had available would not be sufficient to obtain information on MOF 3, but would only obtain a PXRD pattern of MOF 2 . Therefore MOF 2 and 3 were both sent to Los Alamos National Labs and tested and compared at their facilities. Figure S2 in the supplementary data displays the findings of their tests on both samples, and the comparison of the experimental powder patterns and the simulated powder patterns. The comparison clearly shows that the 
major product of the MOF 3 synthesis was MOF 2, while very small peaks located at $2 \theta$ angles of $8^{\circ}$, $12^{\circ}$, and $16^{\circ}$ are all attributed to MOF 3. On the other hand, PXRD patterns of MOF 2 demonstrated that the major product was the MOF 2 structure when compared to the simulated data, both data sets shared major peaks located at $2 \theta$ angles of $10.5^{\circ}, 14^{\circ}, 17^{\circ}$, and $18.5^{\circ}$. PXRD analysis of MOF 4 revealed good crystallinity for the sample, as well as major production of the MOF 4 phase in the sample. Figure S3 shows the comparison of the experimental data obtained for MOF $\mathbf{4}$ and the simulated data, and it can be clearly seen that both data sets have a good correlation with each other.

Thermal Gravimetric Analysis (TGA) measurements were performed only on MOF 2, and 4. Unfortunately there was not enough sample of purified MOF 3 to perform TGA analysis. Figure S4 in the supplementary data show the results of the TGA measurements for MOF 2 . The TGA curve reveals that the water solvent is initially lost at a temperature range of $20-50{ }^{\circ} \mathrm{C}$, then the MOF remains stable until a temperature of approximately $455^{\circ} \mathrm{C}$ at which point the MOF decomposes. Similarly to MOF 2 , MOF 4 also had a similar TGA curve as seen in Figure S5. The methanol solvent is lost between a temperature range of $20-65{ }^{\circ} \mathrm{C}, \mathrm{MOF} 4$ then remains thermally stable until a temperature of about $430{ }^{\circ} \mathrm{C}$, finally decomposing at higher temperatures.

Photoluminescent measurements of both compound 1 and MOF 4 were carried out in the solid state at room temperature (Figure 6). Compound 1 demonstrated a maximum emission wavelength at $365 \mathrm{~nm}$ $\left(\lambda_{\text {ex }}=310 \mathrm{~nm}\right)$, which is the same wavelength given by the powder form of the free $\mathrm{H}_{2} \mathrm{TBC}$ ligand. MOF 4 displayed a maximum emission at $450 \mathrm{~nm}\left(\lambda_{\mathrm{ex}}=380 \mathrm{~nm}\right)$ in the solid state, which was similar to the previously reported emission spectra of MOF $2\left(\lambda_{\mathrm{em}}=432 \mathrm{~nm}\right)$ [12] and $3\left(\lambda_{\mathrm{em}}=438 \mathrm{~nm}\right)$ [8], and can be assigned to the ligand-centered $n-\pi^{*}$ or $\pi-\pi^{*}$ charge transitions. Ethanol changed or thermal treated sample of $\mathbf{4}$ showed same emission spectra at the same excitation wavelength, which also suggested the ligand-centered charge transition mechanism.

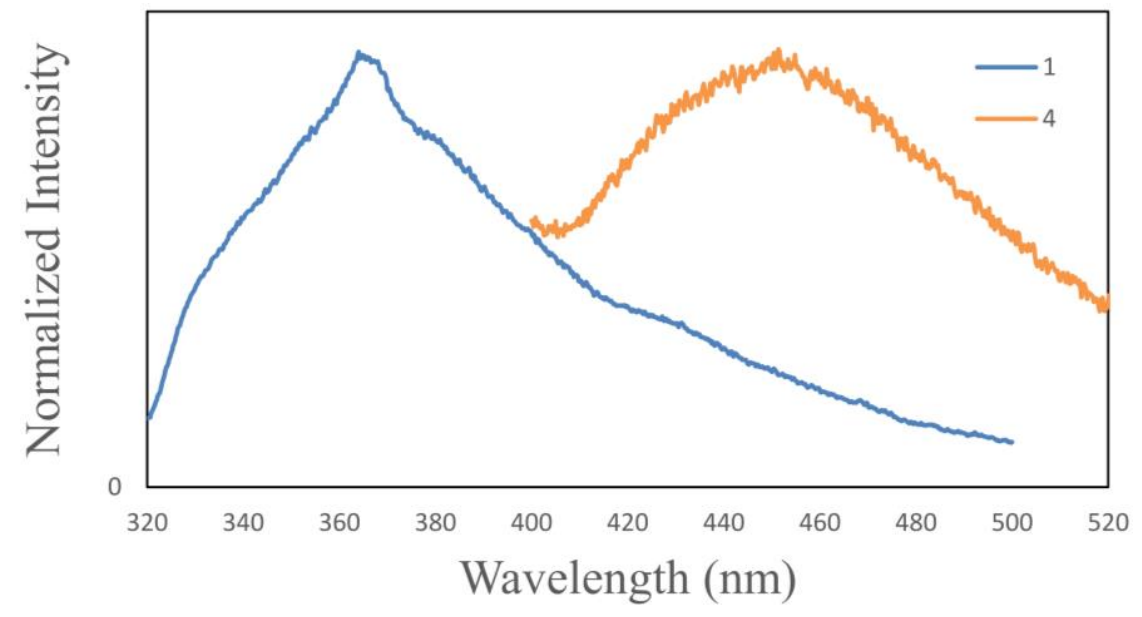

Figure 6. Solid-state emission and excitation spectra of $\mathbf{1}$ and $\mathbf{4}$.

\section{Experimental}

\subsection{Materials and General Methods}

All the solvents and reagents for synthesis were commercially available and used as received. The infrared (IR) spectra were recorded on a Thermo Nicolet 6700 FTIR spectrometer (Thermo Electron 
Corporation, Madison, WI, USA) operating at a spectral resolution of $2 \mathrm{~cm}^{-1}$ accumulating 32 scans. Powder X-ray diffraction (PXRD, Bruker AXS Inc., Madison, WI, USA) of complexes 2 and $\mathbf{3}$ were recorded on a Siemens D-500 X-ray diffractometer operating at $45 \mathrm{kV}$ and $35 \mathrm{~mA}$ and using $\mathrm{Cu} \mathrm{Ka}$ radiation. PXRD patterns of compounds 1 and $\mathbf{4}$ were recorded on a GBC Mini Materials Analyzer powder X-ray diffractometer (GBC Scientific Equipment, Arlington Heights, IL, USA) operating at $35 \mathrm{kV}$ and $30 \mathrm{~mA}$ using $\mathrm{Cu} \mathrm{K \alpha}$ radiation $(\lambda=1.5418 \AA)$. The $2 \theta$ angles were recorded from $5^{\circ}$ to $40^{\circ}$. TGA analyses were measured on an STA7200 Thermal Analysis System with a nitrogen flow of $100 \mathrm{cc} / \mathrm{min}$. The temperature range was from $30{ }^{\circ} \mathrm{C}$ to $500{ }^{\circ} \mathrm{C}$ at a heating rate of $10{ }^{\circ} \mathrm{C} / \mathrm{min}$. Photoluminescence measurements were performed on a Shimadzu RF-5301 PC spectrofluorophotometer with a xenon lamp light source at ambient temperature.

\subsection{Synthesis of 4-Tetrazolyl Benzenecarboxylic Acid}

Sodium azide (364 mg, $5.6 \mathrm{mmol})$, and 4-cyanobenzoic acid (823 g, $5.6 \mathrm{mmol})$ were measured and placed in a beaker and dissolved in a 1:1 solution of ethanol $(25 \mathrm{~mL})$ and water $(25 \mathrm{~mL})$. The solution was sonicated and transferred to a Teflon-lined autoclave, which was placed in the oven at $150{ }^{\circ} \mathrm{C}$ for $24 \mathrm{~h}$. The autoclave was removed from the oven and allowed to cool to room temperature. The white precipitate was then collected into a beaker and washed with deionized water. The precipitate was then transferred to Hirsch funnel and filter flask to remove the water. The collected liquid was then treated with $0.1 \mathrm{M} \mathrm{HCl}$ to precipitate any deprotonated product within the solution. The solution was passed again through the Hirsch funnel and washed with fresh deionized water. The precipitate was then placed in the oven at $150{ }^{\circ} \mathrm{C}$ for $10 \mathrm{~min}$, and then in another oven set at $85{ }^{\circ} \mathrm{C}$ for one hour to completely dry the sample. Yield: $1.0249 \mathrm{~g}(\sim 96 \%)$. FT-IR analysis showed the removal of the cyano groups located at around $2100 \mathrm{~cm}^{-1}$, and the formation of peaks at $1400 \mathrm{~cm}^{-1}$ indicating a tetrazolate group in the structure, and peaks at $1600 \mathrm{~cm}^{-1}$ indicating the existence of the carboxylate group (Figure S6).

\subsection{Crystallization of 4-Tetrazolyl Benzenecarboxylic Acid, 1}

$\mathrm{Zn}\left(\mathrm{NO}_{3}\right)_{2} \cdot 6 \mathrm{H}_{2} \mathrm{O}$ (500 mg, $\left.1.68 \mathrm{mmol}\right)$, and 4-tetrazolyl benzenecarboxylic acid (78 $\left.\mathrm{mg}, 0.419 \mathrm{mmol}\right)$ were measured in a $4: 1$ ration and dissolved in water $(50 \mathrm{~mL})$. The solution was sonicated and transferred to a Teflon-lined autoclave and placed in an oven set at $150{ }^{\circ} \mathrm{C}$ for 3 days. The autoclave was allowed to cool to room temperature. The gel like sample was collected into a capped vial and washed with fresh water. The sample consisted of long hair-like crystals. Inspection via single crystal X-ray crystallography of the sample revealed crystallized 4-tetrazolyl benzenecarboxylic acid.

\subsection{Synthesis of $\mathrm{Zn}_{2}(T B C)_{2}, 2$}

$\mathrm{Zn}\left(\mathrm{NO}_{3}\right) \cdot 6 \mathrm{H}_{2} \mathrm{O}$ (200 mg, $0.673 \mathrm{mmol}$ ), and 4-tetrazolyl benzenecarboxylic acid (150 mg, $\left.0.806 \mathrm{mmol}\right)$ were placed in a vial and dissolved in $10 \mathrm{~mL}$ of water and sonicated until a homogenous solution was formed. The solution was then transferred to a Teflon-lined autoclave and inserted into an oven at $150{ }^{\circ} \mathrm{C}$ for 3 days. The autoclave was removed from the oven and allowed to cool to room temperature for about $3 \mathrm{~h}$. The crystals recovered were washed with fresh water and placed in a vial to be sonicated to remove any powder in the crystal sample. The resulting crystals were yellow fine powder plate crystals. 


\subsection{Synthesis of $\mathrm{Zn}_{2}(\mathrm{TBC})_{2}\left(\mathrm{H}_{2} \mathrm{O}\right), 3$}

$\mathrm{Zn}\left(\mathrm{NO}_{3}\right)_{2} \cdot 6 \mathrm{H}_{2} \mathrm{O}(0.2 \mathrm{~g}, 0.68 \mathrm{mmol})$ and 4-tetrazolyl benzenecarboxylic acid $(0.15 \mathrm{~g}, 0.79 \mathrm{mmol})$ were added into mixed methanol $(5 \mathrm{~mL})$ and water $(5 \mathrm{~mL})$ solution. The mixture was transferred to a Teflon-lined autoclave and placed inside an oven at $150{ }^{\circ} \mathrm{C}$ for 3 days. The autoclave was then placed on a counter for about $3 \mathrm{~h}$ to allow the temperature to decrease to room temperature. The crystals were recovered and washed with a fresh mixture of methanol and water. Only a few crystals of compound $\mathbf{3}$ could be recovered to do single crystal X-ray analysis, the rest of the crystals were made up of MOF 2 crystals.

\subsection{Synthesis of $\left[\mathrm{Zn}_{2}(\mathrm{TBC})_{2}(\mathrm{MeOH})\right] 0.5\left(\mathrm{H}_{2} \mathrm{O}\right), 4$}

$\mathrm{Zn}\left(\mathrm{NO}_{3}\right) \cdot 6 \mathrm{H}_{2} \mathrm{O}(100 \mathrm{mg}, 0.337 \mathrm{mmol})$, and 4-tetrazolyl benzenecarboxylic acid (62 $\left.\mathrm{mg}, 0.333 \mathrm{mmol}\right)$ were measured and dissolved in $10 \mathrm{~mL}$ of methanol. The solution was sonicated until a homogenous solution was achieved. The solution was then transferred to a Teflon-lined autoclave and inserted into an oven at a temperature of $150{ }^{\circ} \mathrm{C}$ for 3 days. The autoclave was then placed on a counter for about $3 \mathrm{~h}$ to allow the temperature to decrease to room temperature. The crystals were then recovered, placed in a vial, washed with fresh methanol, and sonicated to remove any excess powder. The resulsting crystals were white fine powder plate crystals.

\subsection{Computational Calculations}

The Gaussian 09W software (Gaussian Inc., Wallingford, CT, USA) was utilized to optimize the structure and perform the charge distribution calculations. The Gaussview 5.0 program draws the molecular structure of TBC with the charge distribution distinguished by APT color schemes.

\subsection{Crystal Structure Determination}

Single crystals of each compound were selected using a cross-polarized light microscope. The crystal data sets were collected with a Bruker APEX-II CCD diffractometer (Bruker AXS Inc., Madison, WI, USA) using the SAINT+ ver. 6.2 software [18] with graphite-monochromated Mo-K radiation using phi/omega scans. The crystal was mounted onto the diffractometer at a temperature of $100 \mathrm{~K}$ under liquid nitrogen. Three scans were performed with 364 frames for each scan. The structures were solved using the SHELXTL program [19] package and the non-hydrogen atoms were refined with anisotropic thermal parameters. Hydrogen atoms were added at idealized positions, and a riding model was used for subsequent refinement. The function minimized was $\Sigma\left[w\left(|F \mathrm{O}|^{2}-|F \mathrm{c}|^{2}\right)\right]$ with reflection weights $w-1=\left[\sigma^{2}\left|F_{\mathrm{o}}\right|^{2}+\left(g_{1} P\right)^{2}+\left(g_{2} P\right)\right]$ where $P=\left[\max \left|F_{\mathrm{o}}\right|^{2}+2|F \mathrm{c}|^{2}\right] / 3$.

\section{Conclusions}

There are advantages of using hybrid-functional groups' ligands to synthesize multi-topology MOFs, especially when applying structural directing agents to form porous frameworks, such as in the case of framework 4. However, as demonstrated in our study of the reactions of TBC ligands with zinc, it is important to first understand the coordination behaviors of such ligands, especially the coordination modes with metal ions to control the structure of the desired products. In the aqueous and alcoholic 
solvents,

the

"opposite-on" $\mu_{2}-\mathrm{N}(1), \mathrm{N}(4)$ coordination mode of the tetrazolate group of TBC with Zn (II) prevented formation of $\mathrm{Zn}$ clusters to introduce highly opened porous structures. Because of this coordination mode, although the frameworks of all three MOFs are different, the connections of ligands were all through isolated $\mathrm{Zn}$ ions, thus leading to ligand centered charge transitions that can therefore control the fluorescent property of the frameworks. All three MOFs exhibit a similar emission wavelength with maximum intensity of $\sim 440 \mathrm{~nm}$. The influences of the electron donor or acceptor groups on the coordination behaviors of such TBC type ligands are under investigation to expand the coordination mode for the tetrazolate groups. Despite previous reports, this study synthesized framework 2 utilizing $\mathrm{H}_{2} \mathrm{BTC}$ as a precursor and $\mathrm{Zn}\left(\mathrm{NO}_{3}\right) \cdot 6 \mathrm{H}_{2} \mathrm{O}$ as the metal source under hydrothermal conditions. Hence, solvothermal technique served as a potential tool for synthesizing new, and interesting frameworks by just varying the solvent system and keeping other parameters unchanged. This study revealed that simply changing one parameter in the synthetic technique can have significant effects on the formation of certain topologies of MOFs.

Table 1. Crystallographic data and structural refinement summary for complexes 1 to 4 .

\begin{tabular}{|c|c|c|c|c|}
\hline Complex \# & 1 & 2 & 3 & 4 \\
\hline References \# & [16] & {$[7,12,17]$} & {$[8]$} & {$[13]$} \\
\hline Empirical formula & $\mathrm{C}_{8} \mathrm{H}_{6} \mathrm{~N}_{4} \mathrm{O}_{2} \cdot \mathrm{H}_{2} \mathrm{O}$ & $\mathrm{C}_{8} \mathrm{H}_{4} \mathrm{~N}_{4} \mathrm{O}_{2} \mathrm{Zn}$ & $\mathrm{C}_{16} \mathrm{H}_{9} \mathrm{~N}_{8} \mathrm{O}_{5} \mathrm{Zn}_{2}$ & $\mathrm{C}_{8} \mathrm{H}_{6} \mathrm{~N}_{4} \mathrm{O}_{2} \mathrm{Zn}$ \\
\hline Formula weight $(\mathrm{g} / \mathrm{mol})$ & 208.18 & 253.52 & 524.05 & 255.54 \\
\hline Crystal system & Monoclinic & Monoclinic & Hexagonal & Monoclinic \\
\hline Space group & $\mathrm{P} 2_{1} / n$ & $\mathrm{Pc}$ & $R-3 c$ & $\mathrm{C} 2 / c$ \\
\hline$a(\AA)$ & $4.8650(16)$ & $4.8086(11)$ & $26.1408(13)$ & $10.242(4)$ \\
\hline$b(\AA)$ & $5.1946(17)$ & $8.2211(19)$ & $26.1408(13)$ & $34.136(12)$ \\
\hline$c(\AA)$ & $34.110(11)$ & $10.578(3)$ & $13.3926(14)$ & $6.824(2)$ \\
\hline$\alpha\left(^{\circ}\right)$ & 90.00 & 90.00 & 90.00 & 90.00 \\
\hline$\beta\left(^{\circ}\right)$ & $90.592(5)$ & $96.535(3)$ & 90.00 & $115.334(4)$ \\
\hline$\gamma\left({ }^{\circ}\right)$ & 90.00 & 90.00 & 120.00 & 90.00 \\
\hline Volume $\left(\AA^{3}\right)$ & $862.0(5)$ & $415.44(17)$ & $7925.6(10)$ & $2156.4(13)$ \\
\hline$Z$ & 4 & 2 & 18 & 8 \\
\hline Density $\left(\mathrm{Mg} / \mathrm{m}^{3}\right)$ & 1.604 & 2.027 & 1.976 & 1.574 \\
\hline$\mu(\operatorname{Mo~K\alpha })\left(\mathrm{mm}^{-1}\right)$ & 0.127 & 2.936 & 2.777 & 2.263 \\
\hline$F(000)$ & 432 & 252 & 4698 & 1024 \\
\hline Total reflections & 3179 & 4014 & 17689 & 19860 \\
\hline Unique reflections & 684 & 1928 & 2433 & 2595 \\
\hline $\mathrm{R}$ (int) & 0.0234 & 0.0231 & 0.0989 & 0.0718 \\
\hline$R 1[I>2 \sigma(I)]$ & 0.0256 & 0.0257 & 0.0355 & 0.0619 \\
\hline$w R 2[I>2 \sigma(I)]$ & 0.0781 & 0.0633 & 0.0649 & 0.1206 \\
\hline$R 1$ (all data) & 0.0281 & 0.0274 & 0.0747 & 0.0872 \\
\hline$w R 2$ (all data) & 0.0832 & 0.0642 & 0.0743 & 0.1302 \\
\hline GOF on $F^{2}$ & 0.800 & 1.047 & 0.916 & 1.176 \\
\hline
\end{tabular}




\section{Acknowledgments}

This work was funded by the NSF DMR-0934212 (PREM) and EPSCoR IIA-1301346 grants. Some of the work was also supported by the laboratory-directed research and development program of Los Alamos National Laboratory, which is operated by Los Alamos National Security LLC, under DOE Contract DE-AC52-06NA25396.

\section{Author Contributions}

Carlos Ordonez and Tiffany L. Kinnibrugh acquired and analyzed the single crystal X-ray diffraction data sets. Carlos Ordonez performed and analyzed the TGA, fluorescence spectroscopy, IR, and thermogravimetric analysis. Jennifer Lindline and Carlos Ordonez performed and analyzed the powder diffraction X-ray analysis. All authors participated in data interpetration. Tatiana Timofeeva participated in discussion on X-ray structural results and manuscript writing. Qiang Wei and Hongwu Xu designed the experiments. Qiang Wei wrote the manuscript, which was revised and accepted by all the co-authors.

\section{Conflicts of Interest}

The authors declare no conflict of interest.

\section{References}

1. Eddaoudi, M.; Moler, D.; Li, H.; Reineke, T.M.; O’Keeffe, M.; Yaghi, O.M. Modular Chemistry: Secondary Building Units as a Basis for the Design of Highly Porous and Robust Metal-Organic Carboxylate Frameworks. Acc. Chem. Res. 2001, 34, 319-330.

2. Eddaoudi, M.; Kim, J.; Rosi, N.; Vodak, D.; Wachter, J.; O’Keeffe, M.; Yaghi, O.M. Systematic design of pore size and functionality in isoreticular MOFs and their applications in methane storage. Science 2002, 295, 469-472.

3. Moulton, B.; Zaworotko, M.J. From Molecules to Crystal Engineering: Supramolecular Isomerism and Polymorphism in Network Solids. Chem. Rev. 2001, 101, 1629-1658.

4. James, S.L. Metal-organic frameworks. Chem. Soc. Rev. 2003, 32, 276-288.

5. Sudik, A.C.; Millward, A.R.; Ockwig, N.W.; Côté, A.P.; Kim, J.; Yaghi, O.M. Design, Synthesis, Structure, and Gas $\left(\mathrm{N}_{2}, \mathrm{Ar}, \mathrm{CO}_{2}, \mathrm{CH}_{4}\right.$, and $\left.\mathrm{H}_{2}\right)$ Sorption Properties of Porous Metal-Organic Tetrahedral and Heterocuboidal Polyhedra. J. Am. Chem. Soc. 2005, 127, 7110-7118.

6. Pan, L.; Liu, H.M.; Lei, X.G.; Huang, X.Y.; Olson, D.H.; Turro, N.J.; Li, J. A Recyclable Nanoporous Material Suitable for Ship-In-Bottle Synthesis and Large Hydrocarbon Sorption. Angew. Chem. Int. Ed. 2003, 42, 542-546.

7. Song, W.; Li, J.; Song, P.; Tao, Y.; Yu, Q.; Tong, X.; Bu, X. Tuning the Framework Topologies of $\mathrm{Co}^{\mathrm{II}}$-Doped $\mathrm{Zn}^{\mathrm{II}}$-Tetrazole-benzoate Coordination Polymers by Ligand Modifications: Structures and Spectral Studies. Inorg. Chem. 2009, 48, 3792-3799.

8. Li, Y.; Zhong, X.; Zheng, F.; Wu, M.; Liu, Z.; Guo, G. A novel metal-organic framework with bifunctional tetrazolate-5-carboxylate ligand: Crystal structure and luminescent properties. Inorg. Chem. Commun. 2011, 14, 407-410. 
9. Farha, O.K.; Hupp, J.T. Rational Design, Synthesis, Purification, and Activation of Metal-Organic Framework Materials. Acc. Chem. Res. 2010, 43, 1166-1175.

10. Chen, X.-M.; Tong, M.-L. Solvothermal in Situ Metal/Ligand Reactions: A New Bridge between Coordination Chemistry and Organic Synthetic Chemistry. Acc. Chem. Res. 2007, 40, 162-170.

11. Makal, T.A.; Yakovenko, A.A.; Zhou, H.-C. Isomerism in Metal-Organic Frameworks: "Framework Isomers". J. Phys. Chem. Lett. 2011, 2, 1682-1689.

12. Li, Y.; Xu, G.; Zou, W.-Q.; Wang, M.-S.; Zheng, F.-K.; Wu, M.-F.; Zheng, H.-Y.; Guo, G.-C.; Hunag, J.-S. A Novel Metal-Organic Network with High Thermal Stability: Nonlinear Optical and Photoluminescent Properties. Inorg. Chem. Commun. 2008, 47, 7945-7947.

13. Wei, Q.; Yang, D.; Larson, T.E.; Kinnibrugh, T.; Zou, R.; Henson, N.; Timofeeva, T.; Xu, H.; Zhao, Y.; Mattes, B.R. Kinetic hysteresis in gas adsorption behavior for a rigid MOF arising from zig-zag channel structures. J. Mater. Chem. 2012, 22, 10166-10171.

14. Wang, X.S.; Tang, Y.Z.; Huang, X.F.; Qu, Z.R.; Che, C.M.; Hong Chan, P.W.; Xiong, R.G. Syntheses, Crystal Structures, and Luminescent Properties of Three Novel Zinc Coordination Polymers with Tetrazolyl Ligands. Inorg. Chem. 2005, 44, 5278-5285.

15. Li, J.R.; Tao, Y.; Yu, Q.; Bu, X.H. A pcu-type metal-organic framework with spindle $\left[\mathrm{Zn}_{7}(\mathrm{OH})_{8}\right]^{6+}$ cluster as secondary building units. Chem. Commun. 2007, 15, 1527-1529.

16. Li, G.Q.; Wu, A.Q.; Li, Y.; Zheng, F.K.; Guo, G.C. 4-(1H-Tetrazol-5-yl)benzoic acid monohydrate. Acta Cryst. 2008, E64, doi:10.1107/S1600536808019053.

17. Jiang, T.; Zhao; Y.F.; Zhang, X.M. Blue-green photoluminescent 5- and 10-connected metal 5-(4'-carboxy-phenyl)tetrazolate coordination polymers. Inorg. Chem. Commun. 2007, 10, 1194-1197.

18. Bruker. SAINTP + for NT. Data Reduction and Correction Program v. 6.2; Bruker AXS: Madison, WI, USA, 2001.

19. Sheldrick, G.M. SHELXTL NT v. 6.12, Structure Determination Software Suite; Bruker AXS: Madison, WI, USA, 2001.

(C) 2015 by the authors; licensee MDPI, Basel, Switzerland. This article is an open access article distributed under the terms and conditions of the Creative Commons Attribution license (http://creativecommons.org/licenses/by/4.0/). 